\title{
Percutaneous Balloon Compression and Percutaneous Radiofrequency Ablation in Patients with Idiopathic Trigeminal Neuralgia: Management Outcome
}

\author{
Ali R. Hamdan $\left.{ }^{*}{ }^{(}\right)$, Radwan Nouby Mahmoud2 ${ }^{2}$, Mohamad A. Farrag1 \\ ${ }^{1}$ Department of Neurosurgery, South Valley University, Qena, Egypt \\ ${ }^{2}$ Department of Neurosurgery, Assuit University, Assuit, Egypt \\ Email: *Abdallahneuro2010@gmail.com, ^Radwannouby@hotmail.com, m-raof@outlook.com
}

How to cite this paper: Hamdan, A.R., Mahmoud, R.N. and Farrag, M.A. (2019) Percutaneous Balloon Compression and Percutaneous Radiofrequency Ablation in Patients with Idiopathic Trigeminal Neuralgia: Management Outcome. Open Journal of Modern Neurosurgery, 9, 393-400. https://doi.org/10.4236/ojmn.2019.94036

Received: July 2, 2019

Accepted: September 14, 2019

Published: September 17, 2019

Copyright $\odot 2019$ by author(s) and Scientific Research Publishing Inc. This work is licensed under the Creative Commons Attribution International License (CC BY 4.0).

http://creativecommons.org/licenses/by/4.0/

\begin{abstract}
Background: Trigeminal neuralgia is a chronic pain syndrome characterized by paroxysmal, shock-like, stabbing, recurrent episodes of pain localized in the distribution area of one or more branches of the trigeminal nerve. The Initial approach for treating trigeminal neuralgia consists of pharmacological therapy. Many patients experience initial pain relief with pharmacological therapy; however, most of those patients eventually undergo surgery. $\mathrm{Pa}$ tients and Methods: In this descriptive prospective study, we evaluate the efficiency and safety of percutaneous balloon compression (PBC) and percutaneous radiofrequency ablation (RFA) in treating patients with typical idiopathic trigeminal neuralgia. This study includes 21 patients (divided into two groups, $\mathrm{PBC}=15$ patients and $\mathrm{RFA}=$ six patients) who met our inclusion and exclusion criteria, from May 2018 to April 2019, with a follow-up period of six months. Results: At one month postoperative follow-up, all patients in both groups were pain-free. At six months postoperative follow-up, in group 1 (PBC), 14 (95.24\%) patients were pain-free, while in group 2 (RFA) all patients remained pain-free. The most common encountered postoperative complications are masticatory muscles weakness (Four patients in PBC group, and one patient in RFA group) and facial hypothesia (12 patients in $\mathrm{PBC}$ group and four patients in RFA group). Conclusions: In our study, patients with trigeminal neuralgia were not controlled by drug treatment, but percutaneous balloon compression and percutaneous radiofrequency ablation proved to be very effective treatment options, with no major complications.
\end{abstract}

\section{Keywords}

Trigeminal Neuralgia, Percutaneous Balloon Compression, Percutaneous Radiofrequency Ablation 


\section{Introduction}

Trigeminal neuralgia (TN) is a chronic pain syndrome characterized by recurrent attacks of paroxysmal, stabbing, shock-like pain limited to the distribution area of one or more of the trigeminal nerve branches [1]. Diagnosis of TN is mainly clinical, while a definite etiology can be identified by the use of imaging studies in $15 \%$ of cases, usually in the form of vascular nerve compression [2] [3]. Anticonvulsants are typically used as the medical therapy for the first-line treatment of TN, but the guidelines regarding the minimum duration of medical treatment necessary before starting surgical intervention are not standardized yet. Although it was suggested by some reports that trials of at least two anticonvulsant drugs should be applied before an interventional strategy, little evidence exists to support this idea [2] [4]. For Idiopathic trigeminal neuralgia, percutaneous treatments in current use include percutaneous conventional radiofrequency (CRF), percutaneous balloon compression, and percutaneous retrogasserian glycerol rhizotomy [5] [6] [7]. Evaluation of the efficacy and efficiency of $\mathrm{PBC}$ and RFA in the management of idiopathic trigeminal neuralgia is the aim of this study.

\section{Patients and Methods}

This is a prospective, descriptive study that was conducted on 21 patients with typical idiopathic trigeminal neuralgia during the period from May 2018 to April 2019, with a follow-up of 6 months.

Inclusion criteria: Patients with persistent pain due to idiopathic trigeminal neuralgia, with a pain rating, (during the attack) of at least 5 on a Visual Analogue Pain Scale (VAS: "0" no pain, "10" worst possible pain) and non-satisfactory relief and/or intolerable side effects with medical treatment.

Exclusion criteria: Patients with secondary trigeminal neuralgia induced by a tumor of the posterior fossa, stroke, and multiple sclerosis. Patients with a history of previous treatment with invasive treatments, such as RF, balloon compression, destructive chemicals injection, gamma knife, peripheral neurotomy, or microvascular decompression (MVD).

\subsection{Operative Techniques}

\subsubsection{Percutaneous Balloon Compression (PBC)}

The Procedure was done in Supine position under general anesthesia. After induction of general anesthesia, an oblique submental view of foramen ovale was obtained using C-ARM with the head slightly extended and rotated about $15^{\circ}$ to the contralateral side. A mark on the cheek skin was made opposite to the site of the foramen ovale $(2.5-3 \mathrm{~cm}$ lateral to the angle of the mouth). We used a local anesthetic agent (Lidocaine) to infiltrate the site of the cannula insertion. A small skin puncture using a scalpel blade was made to ease the insertion of the 14-gauge cannula.

We then introduced the cannula with its blunt stylet toward the foramen ovale 
under fluoroscopic guidance in the oblique submental view until it engaged the foramen. Then the blunt stylet was replaced with a straight guiding stylet. Then, we changed the C-ARM to the lateral view and further introduced the stylet slowly until it passed just beyond the intersection between the clivus and the petrous bone penetrating the arachnoid in the Meckel's cave, which was confirmed by egressing of the CSF from the cannula.

The stylet was then withdrawn from the cannula and a 4 Fr. Fogarty embolectomy catheter was inserted in the cannula to reach with its tip in the Meckel's cave just beyond the intersection between the clivus and the petrous bone. The balloon was inflated with $1 \mathrm{ml}$ of water-soluble contrast material (Omnipaque), for 1 minute. The shape of the inflated balloon was observed and if the typical pear-shaped balloon was not obtained, we deflated the balloon to reposition the catheter tip and then re-inflated the balloon. We then deflate the balloon and the catheter was withdrawn followed by withdrawal of the cannula itself. Compression of the cheek at the site of cannula insertion was done for about 5 minutes to prevent subcutaneous hematoma formation. The patient was then awakened and discharged to the ward. The facial sensation, corneal reflex, motor power of masticatory muscles, and immediate postoperative pain were examined and recorded.

\subsubsection{Percutaneous Radiofrequency Ablation (RFA)}

The Procedure was done in Supine position, and intravenous short-acting anesthetics (Propofol) associated with local anesthetic infiltration (Lidocaine) at the site of needle insertion were used. A padded oral airway was placed between the patient's jaws and a reference pad, applied to the patient's skin, was connected to the RF generator for patient grounding. For radiological localization of the foramen ovale, we used the same technique used in percutaneous balloon compression.

A 20 gauge RF needle, with $5 \mathrm{~mm}$ active tip was inserted and introduced towards the foramen ovale under fluoroscopic guidance in oblique submental view until it engaged the foramen. Then we changed the C-ARM projection to obtain a lateral view to assessing the location of the needle tip in relation to the clivus profile. The needle's stylet was then removed and the lesioning electrode was inserted into the needle and connected to the RF generator.

After these steps, the patient was awakened and after making sure that the patient was awake and cooperative, we started sensory stimulation at $50 \mathrm{~Hz}$ to ensure precise localization of needle tip according to the affected division of the 5th nerve. During sensory stimulation, the patient was asked to localize the actual site of paresthesia he felt and compare it to the previous complaint. We also tested the motor response of the mandibular division at $2 \mathrm{~Hz}$ by observing the contractions of the masseter muscle.

After detection of the precise location of the needle, the patient was anesthetized again and lesioning was done at a temperature of $70^{\circ} \mathrm{C}$ for 60 seconds. The patient was then awakened and the facial sensation, corneal reflex, motor power 
of masticatory muscles, and immediate postoperative pain were examined and recorded. The patient was then discharged to the ward.

Some factors can affect the efficiency and safety of percutaneous techniques. The Trigeminal depressor response (hypotension and bradycardia) carries a greater risk for patients with cardiovascular disease making it less tolerable to use PBC in such patients. In addition, the inability to tolerate an awake procedure affects the efficiency and safety of using RFA as such patients do not cooperate during localization.

\subsection{Clinical Follow-Up}

Facial sensation, corneal reflex, and motor power of masticatory muscles were examined immediately before discharging the patients to the ward to record any immediate postoperative complications. The length of the pain-free period and recurrence of symptoms were assessed in our outpatient clinic using Visual Analogue Pain Scale (VAS: “0” no pain, “10” worst possible pain) at one month and six months post-operative for each patient.

Patients were followed up in the outpatient clinic to evaluate the length of the pain-free period and recurrence of symptoms at one month and six months post-operative by using Visual Analogue Pain Scale (VAS: “0” no pain, “10” worst possible pain).

\section{Results}

This study was conducted on 21 patients, divided into 2 groups: Group 1 ( $\mathrm{n}=$ 15) was managed using percutaneous balloon compression (PBC) and Group 2 $(n=6)$ was managed using percutaneous radiofrequency ablation (RFA).

Age distribution and Gender incidence:

The age of our patients ranged from 40 to 75 years with mean age $=59.33 \pm$ 9.84 years, and there was no remarkable difference between males and females incidences (47.6\% males vs. 52.40\% females) (Table 1 ).

\subsection{Clinical Presentation}

In Group 1 (PBC), (11/15) 73.33\% of patients had right side symptoms with (7/15) $46.67 \%$ of the patients having combined V2 and V3 affection. The mean duration of the preoperative complaint was 7.4 years with a range of 4 - 15 years.

Table 1. Age distribution and gender incidence of the patients.

\begin{tabular}{ccc}
\hline \multicolumn{2}{c}{ Age } \\
Range & $40-75$ years \\
Mean & $59.33 \pm 9.84$ \\
Gender & No. of patients & $\%$ \\
Male & 10 & $47.60 \%$ \\
Female & 11 & $52.40 \%$
\end{tabular}


In Group 2 (RF), (4/6) 66.67\% of patients had right side symptoms with (4/6) $66.67 \%$ of the patients having V2 affection. The mean duration of the preoperative complaint was 7.17 years with a range of $4-12$ years. These Clinical presentations are summarized in Table 2.

\subsection{Clinical Outcome}

In group 1 (PBC) $13(86.67 \%)$ patients achieved complete initial pain relief while in group $2(\mathrm{RF})$ all patients achieved complete initial pain relief. At 1 month postoperative follow-up, all patients in both groups were pain-free. At 6 months postoperative follow up, in group 1 (PBC) 14 (95.24\%) patients were pain-free, only one patient had a recurrence of symptoms that were controlled by medical treatment and needed no further intervention, while in group 2 (RFA) all patients remained pain-free.

Regarding post-operative complications, transient masticatory muscles weakness was observed in 4 patients $(26.67 \%)$ of group 1 (PBC), and one patient (16.67\%) of group 2 (RFA). In addition, facial hypothesia was found in 12 patients (80\%) of group 1 (PBC), and 4 patients $(66.67 \%)$ of group 2 (RF). These post-operative clinical outcomes are summarized in Table 3 and Table 4.

Table 2. Clinical presentation (Affected side, divisions, and duration of complaint in years) of the studied patients.

\begin{tabular}{|c|c|c|c|c|}
\hline \multirow{3}{*}{ Variables } & \multicolumn{4}{|c|}{ No. of patients } \\
\hline & \multicolumn{2}{|c|}{$\operatorname{PBC}(n=15)$} & \multicolumn{2}{|c|}{ RFA $(n=6)$} \\
\hline & n. & $\%$ & n. & $\%$ \\
\hline \multicolumn{5}{|c|}{ Affected Side } \\
\hline Rt & 11 & $73.33 \%$ & 4 & $66.67 \%$ \\
\hline Lt & 4 & $26.67 \%$ & 2 & $33.33 \%$ \\
\hline \multicolumn{5}{|c|}{ Affected Division } \\
\hline $\mathrm{V} 2$ & 5 & $33.33 \%$ & 4 & $66.67 \%$ \\
\hline V3 & 3 & $20 \%$ & 1 & $16.67 \%$ \\
\hline $\mathrm{V} 2+3$ & 7 & $46.67 \%$ & 1 & $16.67 \%$ \\
\hline \multicolumn{5}{|c|}{ Duration of complaint } \\
\hline Range & \multicolumn{2}{|c|}{$4-15$} & \multicolumn{2}{|c|}{$4-12$} \\
\hline Mean \pm SD & \multicolumn{2}{|c|}{$7.4 \pm 3.70$} & \multicolumn{2}{|c|}{$7.17 \pm 3.54$} \\
\hline
\end{tabular}

Table 3. Pain-free patients during the follow-up periods.

\begin{tabular}{ccccc}
\hline & \multicolumn{4}{c}{ No. of pain-free patients } \\
\cline { 2 - 5 } Follow up period & \multicolumn{2}{c}{ PBC } & RFA \\
\cline { 2 - 5 } & n. & $\%$ & n. & $\%$ \\
\hline 1 month & 15 & $100 \%$ & 6 & $100 \%$ \\
\hline months & 14 & $95.24 \%$ & 6 & $100 \%$ \\
\hline
\end{tabular}


Table 4. Patients with post-operative complications.

\begin{tabular}{cccccc}
\hline & \multicolumn{4}{c}{ No. of cases } \\
\cline { 2 - 5 } Complications & \multicolumn{3}{c}{ PBC } & \multicolumn{2}{c}{ RFA } \\
\cline { 2 - 5 } & n. & $\%$ & n. & $\%$ \\
\hline Masticatory muscles weakness & 4 & $26.67 \%$ & 1 & $16.67 \%$ \\
Facial hypothesia & 12 & $80 \%$ & 4 & $66.67 \%$ \\
\hline
\end{tabular}

\section{Discussion}

Pharmacological therapy is the initial approach for treating trigeminal neuralgia. Carbamazepine is able to reduce the pain by $70 \%$ [8]. Other drugs as amitriptyline and oxcarbazepine have also been used and showed more irregular results [9]. In many patients pharmacological therapy initially relieves pain; however, more than $50 \%$ of patients with trigeminal neuralgia eventually undergo surgery as have been shown in some studies [10].

As for Percutaneous Balloon Compression, many studies reported high rates of initial pain relief. Kouzounias et al. [11] reported $85 \%$ initial pain relief in their study that included 47 patients with a recurrence rate of $64 \%$ within 1.7 years follow up period, while Abdennebi \& Guenane [12] reported $92.7 \%$ initial pain relief and $27.8 \%$ recurrence rate with a follow-up period of $0.5-27$ years. Masticatory muscle weakness and dysesthesias are the most common complications reported in cases treated by PBC. Chen et al. [13] reported dysesthesia (1.5\%), masticatory Weakness (6.2\%) and corneal Sensory Impairment (2.3\%), while Abdennebi \& Guenane [12] reported dysesthesia (2.8\%), masticatory weakness (10.8\%) and corneal sensory impairment (0.9\%).

As regards Percutaneous Radiofrequency ablation (RFA), most of the studies reported very high initial pain relief. Tew et al. [14] reported a 99.4\% initial pain relief and 20\% recurrence rate with a follow-up period of 1 - 21 years. Kanpolat et al. [15] included 1600 patients in his study and reported $97.6 \%$ initial pain relief and $25.1 \%$ recurrence rate with a follow-up period of $1-25$ years. Same as PBC, the most commonly reported complications in cases treated by RFA are masticatory muscle weakness and dysesthesias. Kanpolat et al. [15] reported dysesthesia (1\%), masticatory weakness (4.1\%) and corneal sensory impairment (5.7\%). On the other hand, Tew et al. [14] reported higher rates of complications as dysesthesia (20\%), masticatory weakness (16\%) and corneal sensory impairment (6\%).

In our study, $86.67 \%$ of patients treated with $\mathrm{PBC}$ had complete initial pain relief while $100 \%$ of patients treated with RFA had complete initial pain relief and that goes in agreement with what published in the literature. Regarding the pain-free period, one patient treated with $\mathrm{PBC}$ had a recurrence of symptoms at 6 months Follow up period and was controlled by medical treatment, while all patients treated with RFA remained pain-free at 6 months follow up period.

As regard to postoperative complications in our study, facial hypothesia was 
found in $80 \%$ of patients treated with $\mathrm{PBC}$, and in $66.67 \%$ of patients treated with RF. Transient masticatory muscles weakness was observed in $26.67 \%$ of patients treated with $\mathrm{PBC}$, and in $16.67 \%$ of patients treated with RF.

Limitation of our study is the lack of the long-term follow-up to assess the long term efficacy of both procedures which could be approached in further studies.

\section{Conclusion}

Trigeminal neuralgia is associated with an overall decreased quality of life. PBC and RFA were proved to be effective treatment modalities with no major complications and they are mainly recommended for elderly patients with high operative risk factors or for those young patients who refuse to undergo MVD.

\section{Conflicts of Interest}

The authors declare no conflicts of interest regarding the publication of this paper.

\section{References}

[1] Bennetto, L., Patel, N.K. and Fuller, G. (2007) Trigeminal Neuralgia and Its Management. BMJ, 334, 201-205. https://doi.org/10.1136/bmj.39085.614792.BE

[2] Gronseth, G., Cruccu, G., Alksne, J., Argoff, C., Brainin, M., Burchiel, K., Zakrzewska, J.M., et al. (2008) Practice Parameter: The Diagnostic Evaluation and Treatment of Trigeminal Neuralgia (An Evidence-Based Review): Report of the Quality Standards Subcommittee of the American Academy of Neurology and the European Federation of Neurological Societies. Neurology, 71, 1183-1190. https://doi.org/10.1212/01.wnl.0000326598.83183.04

[3] Zakrzewska, J.M. (2002) Diagnosis and Differential Diagnosis of Trigeminal Neuralgia. The Clinical Journal of Pain, 18, 14-21. https://doi.org/10.1097/00002508-200201000-00003

[4] Tatli, M., Satici, O., Kanpolat, Y. and Sindou, M. (2008) Various Surgical Modalities for Trigeminal Neuralgia: Literature Study of Respective Long-Term Outcomes. Acta Neurochirurgica, 150, 243-255. https://doi.org/10.1007/s00701-007-1488-3

[5] Brown, J.A. (2009) Percutaneous Balloon Compression for Trigeminal Neuralgia. Clinical Neurosurgery, 56, 73-78.

[6] Asplund, P., Blomstedt, P. and Bergenheim, A.T. (2015) Percutaneous Balloon Compression vs Percutaneous Retrogasserian Glycerol Rhizotomy for the Primary Treatment of Trigeminal Neuralgia. Neurosurgery, 78, 421-428. https://doi.org/10.1227/NEU.0000000000001059

[7] Al Khudhairi, D. (2006) Thermocoagulation of Trigeminal Neuralgia by Radiofrequency-Effectiveness and Results. Middle East Journal of Anaesthesiology, 18, 717.

[8] Van Kleef, M., Van Genderen, W.E., Narouze, S., Nurmikko, T.J., Van Zundert, J., Geurts, J.W. and Mekhail, N. (2009) Trigeminal Neuralgia. Pain Practice, 9, 252-259. https://doi.org/10.1111/j.1533-2500.2009.00298.x

[9] Kanpolat, Y., Savas, A., Ugur, H.C. and Bozkurt, M. (2005) The Trigeminal Tract and Nucleus Procedures in Treatment of Atypical Facial Pain. Surgical Neurology, 64, S96-S100. https://doi.org/10.1016/j.surneu.2005.07.018 
[10] Dalessio, D.J. (1982) Trigeminal Neuralgia. Drugs, 24, 248-255. https://doi.org/10.2165/00003495-198224030-00005

[11] Kouzounias, K., Schechtmann, G., Lind, G., Winter, J. and Linderoth, B. (2010) Factors That Influence Outcome of Percutaneous Balloon Compression in the Treatment of Trigeminal Neuralgia. Neurosurgery, 67, 925-934. https://doi.org/10.1227/NEU.0b013e3181eb5230

[12] Abdennebi, B. and Guenane, L. (2014) Technical Considerations and Outcome Assessment in Retrogasserian Balloon Compression for Treatment of Trigeminal Neuralgia. Series of 901 Patients. Surgical Neurology International, 5, 118. https://doi.org/10.4103/2152-7806.137838

[13] Chen, J.F., Tu, P.H. and Lee, S.T. (2011) Long-Term Follow-Up of Patients Treated with Percutaneous Balloon Compression for Trigeminal Neuralgia in Taiwan. World Neurosurgery, 76, 586-591. https://doi.org/10.1016/j.wneu.2011.05.021

[14] Tew, J.M., Morgan, C.J. and Grande, A.W. (2012) Percutaneous Stereotactic Rhizotomy in the Treatment of Intractable Facial Pain. In: Schmidek and Sweet Operative Neurosurgical Techniques. Indications, Methods, and Results, 6th Edition, Elsevier Inc., Amsterdam, 1409-1418.

https://doi.org/10.1016/B978-1-4160-6839-6.10124-8

[15] Kanpolat, Y., Savas, A., Bekar, A. and Berk, C. (2001) Percutaneous Controlled Radiofrequency Trigeminal Rhizotomy for the Treatment of Idiopathic Trigeminal Neuralgia: 25-Year Experience with 1600 Patients. Neurosurgery, 48, 524-534. https://doi.org/10.1097/00006123-200103000-00013 